\title{
MedienPädagogik
}

Zeitschrift für Theorie und Praxis der Medienbildung

Themenheft Nr. 39: Orientierungen in der digitalen Welt Herausgegeben von Bardo Herzig, Tilman-Mathies Klar, Alexander Martin und Dorothee M. Meister

\section{Das Allgemeine der Bildung in der digitalen Welt}

Dan Verständig

\section{Zusammenfassung}

Digitale Bildung stellt einen zentralen Ansatz dar, um eine zeitgemässe Antwort auf die Herausforderungen der Digitalisierung zu formulieren sowie zukunftsorientierte Handlungsfähigkeit und gesellschaftliche Teilhabe zu gewährleisten. Dabei erweist sich das Zusammenspiel von Mensch und digitalen Technologien über einen instrumentell-qualifikatorischen Blick hinausgehend als überaus komplex, was die grundlegende Frage danach aufwirft, wie Bildung im Sinne der Menschwerdung unter den Bedingungen des Digitalen überhaupt gedacht werden kann. Der Beitrag geht der Frage nach, wie sich die besondere Qualität des Digitalen zu einem bildungstheoretisch fundierten Verständnis von Digitalität verhält und welche Bedeutung das Allgemeine im Zusammenspiel von Bildung und Digitalität spielt. Dabei wird das Verhältnis des Besonderen zum Allgemeinen vor dem Hintergrund digital vernetzter Infrastrukturen diskutiert, um so zu Aussagen über die meist unsichtbaren Abhängigkeiten und deren sichtbare Auswirkungen auf Bildung und den Menschen zu diskutieren.

\section{On the general of education in a digital world}

\begin{abstract}
Digital education is a central approach to address the challenges of a digitized world. The relation between human and digital technologies proves to be extremely complex, and going beyond an instrumental view, it reveals a fundamental problem of how to understand education in the sense of becoming human needs and aside from technologydriven efforts. The article explores the needs for general education in particular to foster a better understanding of the digital condition based on education theory. The article discusses the relationship of the particular to the general against the background of digitally networked infrastructures, to gain further insights on the most invisible dependencies of digital technologies and their impact on education and individuals.
\end{abstract}

\section{Einleitung}

Aktuell arbeiten sich verschiedene Bewegungen, Menschen und Gruppen in Diskursen entlang der Digitalisierung ab, in denen es um Fragen der Bildung und der Transformation gesellschaftlicher Strukturen geht. Mit dem Digitalisierungsdiskurs 
werden Forderungen zur radikalen Umstrukturierung von Schule und anderen Bildungseinrichtungen begründet. Man müsse sich den Herausforderungen stellen, vor denen sich technologisch hochentwickelte Gesellschaften sehen. Während die einen von Breitbandausbau und Wi-Fi für Schulen sprechen, fordern andere mehr Medienkompetenz und Medienbildung.

Bereits daraus ergibt sich ein komplexes Diskursfeld, dass keineswegs unproblematisch ist (vgl. hierzu Biermann und Verständig 2020). Digitale Bildung ist in diesem Zusammenhang zum Schlagwort avanciert, um ganz unterschiedliche Forderungen samt Lösungsstrategien abzuleiten und damit den Diskurs um die Fragen der Bildung mehr und mehr zu einem spekulativen Möglichkeitsraum der digitalen Technologien werden zu lassen. Der Diskurs hat sich damit längst von den zentralen Fragen der Bildung losgelöst, in denen es ganz grundlegend um den Menschen und die Frage geht, wie der Mensch als Mensch überhaupt möglich ist.

Der Beitrag greift diese Entkoppelung und Entwicklungen um Bildung in der digitalen Welt auf, indem er nach der besonderen Qualität des Allgemeinen der Bildung unter den Bedingungen der Digitalität fragt. Damit wird ein Problemfeld adressiert, das sich ganz grundlegend mit der individuellen Herstellung von Orientierungsrahmen des Menschen in der Welt befasst (Tenorth 1997; Mittelstraß 2002; Jörissen und Marotzki 2009). Es wird gezielt auf die individuelle Herstellung von Orientierung und die überindividuelle Aushandlung von gesellschaftlichen Werten im Horizont des Digitalen abgestellt, um so die Problemzonen der sich in den letzten Jahren formierenden Diskurse um Bildung in der digitalen Welt weg von technologisch-instrumentellen Fragen hin zu (medien-)anthropologischen Konsequenzen zu führen. Die Auseinandersetzung mit dem Allgemeinen der Bildung verweist auf eine lange Tradition bildungstheoretischer Bemühungen und bildungspraktischer Umsetzungsstrategien zugleich. Das Allgemeine der Bildung wird dabei oft entgegen der speziellen Bildung verortet - eine Lesart, die sich ausgehend von Humboldts Bildungsbegriff historisch manifestiert und bis heute auch in wissenschaftlichen Auseinandersetzungen Bestand hat. Nach Tenorth $(1994,2 f$.) ist die Dichotomie zwischen dem Allgemeinen der Bildung und dem Speziellen jedoch eine Engführung, die weitergehende Problematiken ausklammert, kategoriale Fehlschlüsse nach sich zieht und grundlegend historisch reduktionistisch ist. Es lohnt sich daher, die Hintergründe dieser doch so häufig behandelten Theorielinie genauer anzusehen, denn die allgemeine Bildung ist eng mit der Legitimationsstrategie der höheren Bildung und damit der Einführung des Abiturs und der Allgemeinbildung verwoben. 


\section{Das Allgemeine der Bildung}

Es war Wilhelm von Humboldt, der vor über 200 Jahren die Allgemeinbildung als Kernaufgabe für Schule definierte und damit eine zumindest für den deutschsprachigen Raum folgenreiche Entwicklung angestossen hat (vgl. Böttcher, Heinemann, und Priebe 2019, 6). Humboldt galt die allgemeine Bildung als notwendiges Instrument, um seine humanistische Bildungstheorie zu konturieren, schulische Bildung systematisch zu rahmen und den Bildungsbegriff abzugrenzen.

«Alle Schulen aber, deren sich nicht ein einzelner Stand, sondern die ganze Nation oder der Staat für diese annimmt, müssen nur allgemeine Menschenbildung bezwecken. - Was das Bedürfnis des Lebens oder eines einzelnen seiner Gewerbe erheischt, muss abgesondert und nach vollendetem allgemeinen Unterricht erworben werden. Wird beides vermischt, so wird die Bildung unrein, und man erhält weder vollständige Menschen noch vollständige Bürger» (Humboldt 1920 [1809], 276f.).

Humboldt entfaltete drei Dimensionen der Allgemeinbildung, die sich in der formalen, materialen und nationalen Dimension fassen lassen. Die formale Dimension bezieht sich in erster Linie auf die Ausprägung des Menschen in seiner Individualität und Wechselwirkung zur Welt und thematisiert damit ganz grundlegend das Selbstund Weltverhältnis. Es geht um die innere Formkraft des Menschen in intellektueller, moralischer und ästhetischer Hinsicht. Allgemeine Bildung ist damit zur Grundlage für das Menschsein und die Menschwerdung geworden. Die vielzitierte Aussage von Humboldt, die gleichzeitig Anstoss zur kontroversen Auseinandersetzung gegeben hat und vor dem Hintergrund einer Subjektkonstitution immer noch gibt, ist folgende:

«Der wahre Zweck des Menschen - nicht der, welchen die wechselnde Neigung, sondern welche die ewig unveränderliche Vernunft ihm vorschreibt - ist die höchste und proportionirlichste Bildung seiner Kräfte zu einem Ganzen» (Humboldt 1851, 9).

Ein zentrales Merkmal zur Ausprägung der Kräfte stellen für Humboldt die Sprachen und Einblicke in andere Kulturen dar (vgl. Koller 1997), um die eigene Sicht auf die Welt ins Verhältnis zu setzen und so die Pluralität der Welt zu erfahren. Dörpinghaus (2019, 42f.) weist hierzu darauf hin, dass Humboldt sich mit der Freiheit als erster Voraussetzung einer allgemeinen Bildung, die weder revolutionär erzwungen noch staatlich befohlen werden kann, zur Idee einer Humanität als Allgemeinbildung bekenne. Sie ist nicht auf eine spezifische Zielsetzung oder Tätigkeit hin ausgerichtet und löst sich von äusseren Zwecksetzungen sowie gesellschaftlichen Anforderungen. Was bleibt, ist ganz im Sinne Kants der Verweis auf den Menschen selbst. Bildung wird hierbei nicht als die spezifische Ausbildung oder die Spezialisierung in 
Form einer differenzierten Expertenhaltung gesehen, sondern vielmehr in der Ausgewogenheit und damit in der allgemeinen Form als erstrebenswert verstanden. Erst in der Wechselwirkung mit der Vielfalt der Welt, die in ihrem Reichtum mit der Pluralität der menschlichen Möglichkeiten korrespondiert, finden die im Menschen liegenden Möglichkeiten ihre Aktualisierung. Durch die allgemeine Bildung sollen die Kräfte des Menschen gestärkt, geläutert und geregelt werden; die spezielle Bildung soll dem Menschen die Fertigkeiten verschaffen und ist damit nah an der berufsbezogenen Bildung zu verorten. Für Humboldt folgten die allgemeine und spezielle Bildung gänzlich unterschiedlichen Grundsätzen.

"Das Allgemeine ist in dieser Anbindung gerade nicht vorgegeben, es entsteht vielmehr erst im Verständnis des Besonderen, von Fall zu Fall und durch den Prozess einer verzögernd-distanzierenden Reflexion. Vor diesem Hintergrund gewinnt die Struktur eines reflektierenden Urteilens, das den Horizont des Allgemeinen erst im situativen Verstehen sucht, Bedeutung» (Dörpinghaus 2019, 49).

Gleichzeitig ist die Fokussierung auf Humanität entgegen utilitaristischen Bildungsvorstellungen eine zeitdiagnostische Beobachtung, die später auch durch die kritische Erziehungswissenschaft ganz direkt aufgegriffen wurde, wenn man an den Widerspruch von Freiheit und Bildung bei Heydorn (1979) denkt. In dem Zusammenhang ist Bildung immer in zweierlei Richtungen zu denken: Einerseits auf das Individuum und die dem Menschen zugrundeliegenden Potenziale, andererseits auf gesellschaftliche Rahmenbedingungen, also überindividuelle Aushandlungsprozesse. Damit lässt sich auch an die von Humboldt aufgestellte Differenz von materialer und formaler Dimension anknüpfen. Zwar geht es bei der materialen Dimension im weitesten Sinne um die Inhalte von Lern- und Bildungsprozessen, jedoch fokussiert Humboldt hierbei das Verhältnis von Form und Stoff vor dem Hintergrund des Selbstund Weltverhältnisses, welches er als Wechselbeziehung zwischen «Ich» und «Welt» fasst. Das ist insofern von gesteigerter Bedeutung, als dass es nach Blankertz (1982, 104) damit auch um die Form der Vermittlung von Lerninhalten geht. Formale und materiale Bildung sind somit lediglich einzelne Facetten eines komplexen Zusammenspiels von Welterkundung. Legt man dies in normativer Lesart strikt aus, dann folgt daraus nicht nur, dass sich Form und Stoff bedingen, es lässt auch den Schluss zu, dass nicht alle Inhalte der Form kompatibel sind und umgekehrt. ${ }^{1}$ Die nationale Dimension und die damit einhergehenden Forderungen sind schliesslich eng an die vorherrschenden gesellschaftlichen Rahmenbedingungen geknüpft. Humboldt beschreibt damit im Kern, dass der neuhumanistische Bildungsgedanke keine Elitenbildung sein kann und formuliert damit die Abkehr von der Standeserziehung, indem er Bildung reformierend als Bildung für die ganze Nation beschreibt.

1 Dieser Aspekt ist insbesondere im Hinblick auf die digitale Bildung von gesteigerter Bedeutung, wie ich nachfolgend ausführen werde. 
Das Allgemeine der Bildung ist damit mehr als nur die curriculare Einbettung von Inhalten zur Förderung von Allgemeinbildung, die ohnehin auf einen Kanon aufbaut, der unter den Bedingungen der Digitalität selbst infrage gestellt wird. Es handelt sich damit um eine immer wiederkehrende Erscheinung der Auseinandersetzungen und der Aktualitätsbezogenheit, ähnlich wie sie mit dem Bildungsbegriff vorgenommen wird. Die Komplexität ergibt sich aus dem Umstand, dass hier einerseits auf die individuelle Verfasstheit des Menschen und seiner Möglichkeiten der Entfaltung abgezielt wird, andererseits eine individuelle Entfaltung jedoch nicht ohne die Berücksichtigung überindividueller Zusammenhänge - also gesellschaftlicher, politischer und kultureller Einflussbereiche - gedacht werden kann. Folgt man Dörpinghaus $(2019,35)$, so bricht die Diskussion um das Allgemeine der Bildung,

«[...] als Diskursfeld zumeist in Krisensituationen auf, sei es, weil neue Moden, Technologien, Wissens- oder Kompetenzansprüche an Gesellschaft und Kultur herangetragen werden, politische Fundamente der Sicherung bedürfen, Chancengleichheiten eingefordert oder grundsätzlich schulische Leistungen problematisiert werden» (ebd.).

Diese Diskurswellen sind zyklisch und keineswegs etwas Neues. Vielmehr ist es gewissermassen eine schon dem Bildungsbegriff zugrundeliegende Komplexität, die zu diesen wiederkehrenden Aktualisierungstendenzen führt. Es handelt sich damit im Kern um ein dem aus dem deutschen Idealismus hervorgegangenen Bildungsbegriff immanentes Problem, das sich in Anlehnung an Sesink (2006, 9) auf die Diskrepanz von Ideal und Praxis zurückführen lässt. Dementsprechend ist es naheliegend, dass Bildung ganz grundlegend auf die Beschaffenheit unter zeitgenössischen Rahmenbedingungen be- und hinterfragt werden kann (vgl. Menze 1965; Klafki 1985; Böttcher, Heinemann, und Priebe 2019). Mit Blick auf die Digitalisierungstendenzen der letzten Jahre und deren Umbrüche in Gesellschaft, Politik und Wissenschaft liegt es nahe, dass Facetten und Ausprägungen des Bildungsgedankens heute erneut infrage gestellt oder zumindest auf Aktualisierung befragt werden.

Entscheidend für das Allgemeine der Bildung ist das Differenzbewusstsein, also die Herausbildung einer Haltung zur Welt, bei der man zwischen richtig und falsch unterscheiden und Orientierung herstellen kann. Hierbei spielen selbstverständlich zeitgenössische Rahmenbedingungen eine wichtige Rolle. Zu fragen gilt es daher, welche Rolle die digitalen Technologien hier einnehmen. Es geht dabei um die übergeordnete Frage danach, wie der Mensch als Mensch überhaupt möglich ist. Es wird somit ganz gezielt auf eine doppelte Differenz abgezielt, die dem Konzept von Bildung zugrunde liegt, in dem es einerseits um die individuelle Herstellung von Orientierung und andererseits die überindividuelle Aushandlung von gesellschaftlichen Normen und Werten geht. 


\section{Bildung und Digitalität}

Mit der übergeordneten Zielsetzung, das Digitale begreifbar, verstehbar und dessen oftmals implizite sowie unsichtbare Auswirkungen auf das Soziale sichtbar zu machen, werden Initiativen, Plattformen und Foren geschaffen, die sich dem meist niederschwelligen Coding verschreiben und dazu verhelfen sollen, formalisierendes Denken, Problemlösungsstrategien im Umgang mit digitalen Technologien zu fördern und so die notwendigen Skills für das 21. Jahrhundert aufzubauen. Dabei herrscht derzeit kaum Konsens über eben Skills und deren Reichweiten.

Bei der Vielzahl dieser Ansätze, die vielerorts Anklang finden, stellt sich dennoch die grundlegende Frage nach den daraus entstehenden Potenzialen für das Allgemeine der Bildung, die sich unter anderem in der Herstellung von Orientierungsrahmen beschreiben lassen. Allerdings kommt es dabei nicht selten zu einer reduktionistischen Verwendung der Termini und ihrer Bedeutungsgehalte: Digitale Bildung ist beispielsweise in recht kurzer Zeit vom Schlagwort zum Megatrend avanciert. In der Konsequenz wird durch eine derartige Zuspitzung auf digitale Technologien nicht nur die Komplexität von Bildung, sondern auch die besondere Charakteristik des Digitalen weitestgehend heruntergebrochen auf den funktionalen Umgang mit spezifischen digitalen Artefakten, Umgebungen und Systemen.

Dies ist der Fall, wenn es einerseits lediglich um den Einsatz digitaler Technologien zur Unterstützung bzw. Verbesserung von Lehr- und Lernprozessen (z.B. in der Schule oder Hochschule) und weniger um Fragen der Orientierung und der Kompetenzentwicklung in einer zunehmend digitalisierten Lebenswelt geht. Andererseits wird bezogen auf den Einsatz digitaler Medien im Bildungsbereich (z.B. beim DigitalPakt) vor allem über die erforderliche technische Ausstattung und weniger über pädagogisch-didaktische Nutzungskonzepte sowie die Fort- und Weiterbildung der Lehrenden diskutiert. Das Problem einer solchen Betrachtung liegt darin, dass bestehende Ordnungen mit neuen technischen Infrastrukturen angereichert werden. Ein Problem ist dies insofern, als dass sich aus dem Zusammenspiel der digitalen Technologien und kulturellen wie auch sozialen Praktiken nicht nur eine Transformation bestehender Ordnungen abzeichnet, sondern gar neue Relationen hervorgehen. Berücksichtigung finden die Transformationsprozesse in einer strukturtheoretischen Perspektive auf Bildung oder - historisch nach Humboldt gerahmt - in der formalen Dimension, die mediale Strukturen als konstitutives Merkmal einer digitalen Welt verstehen.

Mit der Strukturalen Medienbildung haben Jörissen und Marotzki (2009) ein Konzept vorgestellt, das sich im Schnittfeld von Medien- und Bildungstheorie befindet, den Bedingungen von Bildung in einer medial unhintergehbaren Welt Rechnung trägt und dabei weniger die schulische Bildung als vielmehr die Verfasstheit des Menschen allgemein betrachtet. Massgebend für den Gedanken der Medienbildung ist mithin 
«der Umstand, dass erstens Artikulationen von Medialität nicht zu trennen sind und dass zweitens mediale Räume zunehmend Orte sozialer Begegnungen darstellen, dass also mediale soziale Arenen in den Neuen Medien eine immer größere Bedeutung für Bildungs- und Subjektivierungsprozesse einnehmen» (Jörissen und Marotzki 2009, 39).

Medialität meint dabei das komplexe Zusammenspiel verschiedener Medien und deren Implikationen auf das Soziale. Während einem früher viele Entscheidungen durch die Einbettung in traditionale Muster abgenommen wurden, sind die Menschen heute mehr denn je auf sich selbst zurückgeworfen und müssen ihre Optionen selbst finden, bestimmen und nach ihren Massgaben handeln. Der Mensch muss Entscheidungen treffen und kann die auch immer wieder anders treffen. Bildung als Antwort auf diese Situation ist genau das: die Reflexion dieser Zusammenhänge. Wenn es nun im Kern um das Selbst- und Weltverhältnis des Menschen geht, dann schliesst das die Fragen ein, wie wir die Welt um uns herum einerseits wahrnehmen und andererseits gestalten. Die Folge einer solchen Betrachtung eröffnet entgegen einer instrumentell-qualifikatorischen Sichtweise die Hinwendung zur Frage, wie der Mensch als Mensch unter dem Vorzeichen des Digitalen überhaupt möglich ist.

In seinem Band «Kultur der Digitalität» nimmt der Kulturwissenschaftler Stalder (2016) spätmoderne Gesellschaftsformationen in den Blick, die durchzogen sind von digitalen Technologien. Der Begriff der Digitalität knüpft an die Phänomene der Medialität nach Jörissen und Marotzki (2009) insofern an, als dass es hier weniger um technische Betrachtungen, sondern vielmehr um soziale und interaktionsbezogene Aushandlungen geht. Für Stalder ist die Digitalität ein Zustand, der erst dann eingetreten ist, wenn «die Faszination für die Technologie abgeflaut ist und ihre Versprechungen hohl klingen» (Stalder 2016, 20). Erst dann werde die Kultur und Gesellschaft in einem umfassenden Sinne durch Digitalität geprägt (vgl. ebd.). Dabei ist die Kultur der Digitalität die Folge eines «weitreichenden gesellschaftlichen Wandels, dessen Anfänge teilweise bis ins 19. Jahrhundert zurückreichen» (ebd., 16f.). Digitalität meint nicht nur den Umgang mit digitalen Technologien, sondern ein Verhältnis zur Welt, in dem die digitalen Technologien ein konstitutives Merkmal darstellen. Dabei seien die Nutzer in der dynamischen Welt der Unübersichtlichkeit «von einem radikalen, kurzfristigen Pragmatismus» (ebd., 195) geleitet. Bei der Herstellung von individuellen Orientierungsrahmen werden sie abhängig von digitalen Technologien. Gleichzeitig liege die Teilhabe am Gemeinschaftlichen in der Kultur der Digitalität nicht alleine in der Kompetenz des Individuums, da das Individuum auf die Gemeinschaft verwiesen ist (vgl. Allert und Asmussen 2017, 63). Das heisst, dass Praktiken basierend auf einer Kultur der Digitalität immer auch Aushandlungen mit Unbestimmtheit darstellen. 
Dem Digitalen ist schliesslich eine besondere Qualität eingeschrieben, die es aufgrund der weitreichenden Implikationen für die Fragen der Bildung genauer zu betrachten gilt. Dies ist in doppelter Weise zu verstehen, denn einerseits verändern digitale Architekturen und deren Infrastrukturen die Weisen, wie wir Welt wahrnehmen, Kommunikation, Arbeit und Leben gestalten in nicht zu vernachlässigendem Masse; andererseits verändert sich das Digitale kontinuierlich selbst. Veränderungen liegen dem Digitalen gewissermassen als inhärente Eigenschaft zugrunde. Dabei ist die Grammatik des Digitalen hochgradig ambivalent, denn einerseits beruht alles auf den Zeichen 0 und 1 , andererseits kann aus diesen Zeichen, den damit verbundenen abstrahierten Prozessen und Symboliken eine Vielzahl von Entitäten, medialen Erscheinungen und strukturgebenden Formaten hervorgehen. Gleichzeitig können sich diese Strukturen, wie auch die Inhalte, jederzeit wieder neu anordnen und verändern, sie tun dies in scheinbar natürlicher Weise und in enger Verflechtung zu sozialen, kulturellen und politischen Praktiken. Das Digitale, also digitale Technologien und Medien sind damit die Öffnung zum Allgemeinen und gleichzeitig die Ermöglichung des Besonderen.

\section{Das Allgemeine und das Besondere}

Es ist das Verhältnis von Allgemeinem und Besonderen, das schon hinsichtlich des Bildungsbegriffs mit Dörpinghaus (2019) herausgestellt und mit Jörissen und Marotzki (2009) sowie Stalder (2016) akzentuiert wurde. In strukturtheoretischer Perspektive ergeben sich für die individuellen (Such-)Bewegungen zwischen dem Allgemeinen und dem Besonderen weitere Konsequenzen, nimmt man die soziologische Perspektive von Reckwitz (2017) und seine historische Einordnung hinzu:

"Seit dem Ende des 20. Jahrhunderts wechselt die avancierteste Technologie der Epoche nun jedoch gewissermaßen die Seite und verkehrt sich von einem Transformationsriemen der Rationalisierung in einen der Kulturalisierung. Von einem Motor der Standardisierung in einen der Singularisierung» (Reckwitz 2017, 228).

In diesem Zusammenhang ist die soziologische Doppelläufigkeit bei Reckwitz von einiger Bedeutung, denn die Singularisierung und Ausprägung des Individuellen ist nicht mit einer Loskoppelung von gesellschaftlichen Strukturen zu verwechseln. Die Ausprägung von Singularitäten, das Streben nach dem Besonderen, der Drang nach Individualität und die Wege der Selbstentfaltung liegen innerhalb gesellschaftlicher Strukturen und sind stets von diesen bedingt. So lässt sich auch die von Simmel beschriebene Dorfgemeinschaft lesen, bei der sich ein Dorf von einem anderen dahingehend abgrenzt, als dass es sich individualisiert. Wichtig für die hier hervorgebrachte Bedeutung des Digitalen ist allerdings ein anderer Punkt: «Die 
digitalen Technologien nehmen den Stellenwert einer allgemeinen Infrastruktur zur Fabrikation von Singularitäten an» (Reckwitz 2017, 229).

«Das heißt: Im Innern des technologischen Systems herrschen (auch) Verfahren und Formate der Standardisierung und Universalisierung, aber diese bilden die Hintergrundstruktur für die Fabrikation von Singularitäten. Als Infrastruktur erhält die Logik des Allgemeinen damit einen instrumentellen Stellenwert. Sie ist eine Funktion der (maschinellen und kulturellen) Singularisierung und der Etablierung einer globalen Kulturmaschine» (ebd.).

Digitale Technologien und digitale Medien sollten mit Blick auf die Thesen von Lev Manovich $(2001 ; 2013)$ und die Frage der Kreativitätsentfaltung nicht gleichgesetzt werden. Digitale Technologien bringen digitale Medien hervor, die es uns heute leichter machen denn je, kreativ zu sein, mediale Strukturen anzuordnen und neu zu bestimmen. Es ist also möglich, Dinge von einem Format in ein beliebiges anderes Format zu konvertieren. Die Frage, wie digitale Technologien dies ermöglichen, führt jedoch eine andere Richtung als die Frage, welche Formen aus den digitalen Medien in Form medialer Artikulation hervorgehen können. Die Differenz liegt in den kulturellen Ausprägungen oder der Kultur der Digitalität (Stalder 2016).

Was heisst das nun für die Fragen der Bildung? In der Konsequenz ist das Programmieren hier die Ausbildung des Speziellen: Nicht jede und jeder wird programmieren können, so wie nicht jede und jeder die alten Sprachen lernt. Gleichzeitig birgt die Ausprägung der partikularen Bildung die Gefahr einer Halbbildung (vgl. Grimm 1877/2005). Die Fokussierung auf digitale Kompetenzen oder Digital Skills kann dabei neue Distinktionsprozesse hervorbringen, ohne dabei Differenzbewusstsein zu schärfen.

Der Mensch entfaltet seine besondere Ausprägungskraft durch die Digitalen Medien, die aus den digitalen Technologien hervorgehen. Gleichzeitig - und hier liegt ein bildungstheoretisch relevanter Kern - macht das Subjekt sich, um es mit Hegel zu sagen, «allgemein», indem es soziale Vermittlungen eingeht. Dabei macht sich das Subjekt jedoch nie vollkommen allgemein, sondern oszilliert in verschiedenen diffusen Formationen zwischen dem Allgemeinen und dem Besonderen, ohne sich vom einen oder vom anderen komplett loszulösen. Dabei handelt es sich um einen schmalen Grat oder Balance-Akt, der fragil ist und jederzeit in Modi der Bestimmtheitsherstellung münden und dabei die Anerkennung von Unbestimmtheit ausblenden kann. Das heisst aber auch, dass Freiheit als Modus der Selbstbeschreibung und Erkundung sowie Wahrnehmung von Welt immer auch von äusseren Einflüssen bestimmt ist. Das heisst auch, dass Werte in die uns umgebenden digitalen Architekturen eingeschrieben sind und die Komplexität des Zusammenspiels dieser Architekturen eben nicht hinreichend erfasst werden kann, wenn man sich lediglich der Vermittlung von Algorithmen und Datenstrukturen widmet. Reckwitz (2017) beschreibt die soziale Logik 
des Allgemeinen als «eine rein innermaschinelle, insofern technische Logik im engeren Sinne» (ebd., 230). Die von ihm beobachteten Strukturveränderungsprozesse lassen sich in verschiedener Hinsicht auf die Fragen der Bildung übertragen, denn sich im hegelschen Sinne «allgemein» zu machen, ist ein zentrales Moment in der Suchbewegung des Menschen und damit ein unverzichtbarer Bestandteil des häufig umstrittenen Subjektkerns. Auch Humboldt ist nicht vom Menschen als Subjekt ausgegangen, wie einführend erinnert wurde. Zugleich ist mit dem Allgemeinen in dieser Form eine Standardisierung und Konformisierung des Technischen gemeint und eben nicht die allgemeine und möglichst weite Ausprägung der menschlichen Kräfte in alle Richtungen, wie es damals noch von Humboldt hinsichtlich der allgemeinen Bildung formuliert wurde.

Will man sich der Komplexität der Bildung in einer digitalen Welt zuwenden, scheint es sinnvoll, dies über die Frage nach dem Differenzbewusstsein zu machen, die dem Allgemeinen der Bildung verhaftet ist. Das heisst in der Konsequenz, dass man sich den sozialen Wirkweisen digital vernetzter Architekturen zuwendet und die Frage nach den Werten stellt, die in die digitalen Technologien eingeschrieben sind. Eine solche Hinwendung wird besonders dann relevant, wenn man sich den ökonomischen Tendenzen des Netzes widmet. Zuboff (2019) spricht in diesem Zusammenhang und vor dem Hintergrund ihrer Theorie des Überwachungskapitalismus von einer radikalen Indifferenz, die durch die digitalen Technologien und kommerziellen Interessen provoziert wird:

«Radical indifference leaves a void where reciprocities once thrived. For all their freedom and knowledge, this one void that surveillance capitalists will not fill because doing so would violate their own logic of accumulation» (Zuboff 2019, 352).

Zuboff macht in diesem Zusammenhang ganz explizit deutlich, dass die Idee von Humanität, wie sie vor allem im Sinne der hier entfalteten allgemeinen Bildung als Selbstzweck des Menschen gefasst wird, durch die radikale Indifferenz unterwandert wird:

«Instrumentarianism's radical indifference is operationalized in Big Other's dehumanized methods of evaluation that produce equivalence without equality. These methods reduce individuals to the lowest common denominator of sameness - an organism among organisms - despite all the vital ways in which we are not the same» (Zuboff 2019, 354).

Diese Entwicklung pointiert die Notwendigkeit der Rückbesinnung auf das Allgemeine der Bildung in einer digitalen Welt insofern, als dass die Akzeptanz dieser Systeme ganz grundlegend das infrage stellen lassen, was Freiheit bedeutet. Allgemeinbildung verweist somit auf 
«Verwendungskontexte, die sich um die Achsen des Wissens und der Weltdeutung, des Humanismus sowie der Formen von Subjektivität oder auch der kollektiven Identität und Gleichheit drehen» (Dörpinghaus 2019, 51).

Es sind diese Suchbewegungen zwischen dem Allgemeinen und dem Besonderen, die eben nicht nur auf die individuelle Ermächtigung und Kompetenzförderung zielen, sondern stets im Zusammenhang gesellschaftlicher, ökonomischer und politischer Systeme zu denken sind.

\section{Fazit}

Der Beitrag hat den Schwerpunkt auf das Allgemeine der Bildung gelegt, um deutlich zu machen, welche Komplexität mit der Konzeption von Bildung in einer digitalen Welt einhergeht. Digitale Technologien im Zusammenspiel mit sozialen, kulturellen Praktiken verändern gesellschaftliche Entwicklungsprozesse in besonderem Masse. Wenngleich es verlockend scheint, digitale Bildung als Lösungsansatz für die Problemzonen der Allgemeinbildung zu formulieren, geht damit die Gefahr einer technikdeterministischen Sichtweise einher, die es zumindest reflexiv-analytisch einzuholen gilt. Digitale Technologien lassen sich aus der heutigen Welt nicht mehr hinausdividieren und die aus ihnen hervorgehenden digitalen Medien bieten enormes Potenzial, um Lern- und Bildungsprozesse nicht nur anzureichern, sondern grundlegend die Art und Weise im Umgang mit Wissen zu verändern. Die Potenzialentfaltung ist jedoch kein Selbstläufer. Vielmehr zeigt sich vor dem Hintergrund der hier geführten Diskussion um das Allgemeine der Bildung, dass die Komplexität einer digitalen Welt keineswegs nur auf technische Aspekte zu reduzieren ist. Unter Zuhilfenahme der soziologischen Perspektive mit Reckwitz (2017) konnten Mechanismen der Kulturalisierung der Gesellschaft in Beziehung zu den digitalen Technologien gesetzt werden. Die individuellen Suchbewegungen zwischen dem Allgemeinen und dem Besonderen sind bildungstheoretisch hochgradig relevant, da sie die Frage nach der Herstellung von Orientierung adressieren und mit Blick auf die digitalen Medien auch empirisch erfassbar erscheinen lassen. 


\section{Literatur}

Allert, Heidrun, und Michael Asmussen. 2017. «Bildung als produktive Verwicklung». In Digitalität und Selbst, hrsg. v. Heidrun Allert, Michael Asmussen, und Christoph Richter. Bielefeld: transcript. https://doi.org/10.14361/9783839439456-004.

Biermann, Ralf, und Dan Verständig. 2020; im Erscheinen. «Digitalisierung und Schule: Medienbildung und -kompetenz als schulische Zielvorstellung». In Medienbildung und Schulkultur in der Reihe Schule und Gesellschaft. hrsg. v. Claudia Kuttner und Stephan Münte-Goussar, Wiesbaden: Springer VS.

Blankertz, Herwig. 1982. Die Geschichte der Pädagogik: von der Aufklärung bis zur Gegenwart. Wetzlar: Büchse der Pandora.

Dörpinghaus, Andreas. 2019. «Die Grammatik der Allgemeinbildung». In Allgemeinbildung im Diskurs. Plädoyer für eine Kernaufgabe der Schule, 35-53. Hannover: Friedrich Verlag.

Grimm, Jacob, und Wilhelm Grimm. 1877/1984. «Halbbildung». In Deutsches Wörterbuch. Band 10. Leipzig.

Heydorn, Heinz-Joachim. 1979. Über den Widerspruch von Bildung und Herrschaft: Bildungstheoretische Schriften Band 2. Frankfurt am Main: Syndikat.

Humboldt, Wilhelm von. 1920. Gesammelte Schriften (Bd. XIII). Berlin: B. Behr.

Humboldt, Wilhelm von, und Clemens Menze. 1997. Bildung und Sprache. 5., durchges. Aufl. Schöninghs Sammlung pädagogischer Schriften. Paderborn: Schöningh.

Jörissen, Benjamin, und Winfried Marotzki. 2009. Medienbildung - Eine Einführung: Theorie Methoden - Analysen. UTB Erziehungswissenschaft, Medienbildung 3189. Bad Heilbrunn: Klinkhardt.

Klafki, Wolfgang. 1985. Neue Studien zur Bildungstheorie und Didaktik: Beiträge zur kritischkonstruktiven Didaktik. Weinheim: Beltz.

Koller, Hans-Christoph. 1997. «Bildung in einer Vielfalt der Sprachen: Zur Aktualität Humboldts für die bildungstheoretische Diskussion unter den Bedingungen der (Post-) Moderne». In Die Zukunft des Bildungsgedankens, hrsg. v. Lutz Koch und Walter Bauer, 45-64. Schriften zur Bildungs- und Erziehungsphilosophie. Weinheim: Deutscher Studien Verlag.

Manovich, Lev. 2001. The language of new media. Cambridge, Mass.: MIT Press.

Manovich, Lev. 2013. Software Takes Command. International Texts in Critical Media Aesthetics. London: Bloomsbury.

Menze, Clemens. 1965. Wilhelm von Humboldts Lehre und Bild vom Menschen. Ratingen: Henn.

Mittelstraß, Jürgen. 2002. «Bildung und ethische Maße». In Die Zukunft der Bildung, hrsg. v. Nelson Killius, 151-170. Frankfurt am Main: Suhrkamp.

Reckwitz, Andreas. 2017. Die Gesellschaft der Singularitäten: zum Strukturwandel der Moderne. Berlin: Suhrkamp.

Stalder, Felix. 2016. Kultur der Digitalität. Edition Suhrkamp, Bd. 2679. Berlin: Suhrkamp.

Zuboff, Shoshana. 2019. The Age of Surveillance Capitalism: The Fight for the Future at the New Frontier of Power. London: Profile Book. 